\title{
Interbands phase models for polychromatic image reconstruction in optical interferometry
}

\author{
Antony Schutz ${ }^{\mathrm{a}}$, André Ferrari ${ }^{\mathrm{a}}$, Éric Thiébaut ${ }^{\mathrm{b}}$, Férréol Soulez $^{\mathrm{b}, \mathrm{c}}$, \\ Martin Vannier ${ }^{\mathrm{a}}$, and David Mary ${ }^{\mathrm{a}}$ \\ ${ }^{a}$ Lab. J.-L. Lagrange, Université de Nice Sophia Antipolis, CNRS, Observatoire de la Côte \\ d'Azur, Parc Valrose, F-06108 Nice cedex 02, France

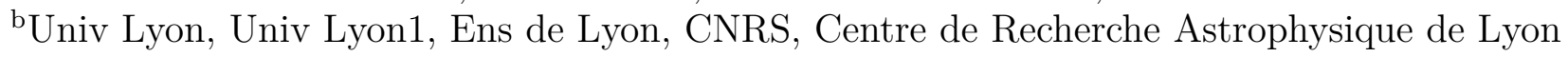 \\ UMR5574, F-69230, Saint-Genis-Laval, France \\ ${ }^{ }$Biomedical Imaging Group, École polytechnique fédérale de Lausanne (EPFL), Lausanne \\ CH-1015, Switzerland
}

\begin{abstract}
This paper presents an extension of the spatio-spectral ("3D") image reconstruction algorithm called PAINTER (Polychromatic opticAl INTErferometric Reconstruction software). The algorithm is able to solve large scale problems and relies on an iterative process, which alternates estimation of polychromatic images and of complex visibilities. The complex visibilities are not only estimated from squared moduli and closure phases, but also from differential phases, which helps to constrain the polychromatic reconstruction. Alternative methods to construct the specific differential phases used in PAINTER are proposed. Simulations on synthetic data illustrate the specificities of the proposed methods.
\end{abstract}

Keywords: Image reconstruction, multiwavelengths, phases estimation, optical interferometry

\section{INTRODUCTION}

Polychromatic OI image reconstruction has recently became a very active domain of research. A spatio-spectral image reconstruction algorithm named PAINTER ${ }^{1,2}$ (for Polychromatic opticAl INTErferometric Reconstruction software) with a publicly available source code* has been proposed. This algorithm relies on the visibilities $\left(\gamma^{2}\right)$ as measures of the source power spectrum and on two types of phases differences: the phase closures $(\psi)$ at each wavelength which are turbulence independent and the differential phases, ${ }^{3}$ which are defined as the phases relatively to a reference channel at each base. The objective of this communication is to present a number of improvements brought to the prototype version of PAINTER algorithm. These improvements $i$ ) concern the acceleration and the conditioning of the algorithm and ii) to propose alternative methods to construct the differential phases used by the algorithm.

\section{MODEL FOR PHASE RELATIONSHIPS}

In the presence of atmospheric turbulence the beams received at each telescope are affected by random and different optical paths which corrupt the phases measurements of the complex visibilities. The "atmospheric corrupted" visibilities at a given wavelength $\lambda_{n}$ for the base $\mathbf{b}_{a, b}$ involving telescopes $a$ and $b$ can be modeled as:

$$
\mathrm{y}_{a, b}^{\lambda_{n}}=\gamma_{a, b}^{\lambda_{n}} \exp \left(\mathrm{i}\left[\varphi_{a, b}^{\lambda_{n}}+\eta_{a}^{\lambda_{n}}-\eta_{b}^{\lambda_{n}}\right]\right)
$$

where $\varphi^{\lambda_{n}}$ is the uncorrupted phases and $\eta^{\lambda_{n}}$ are perturbation terms related to telescopes $a$ and $b$. To overcome the difficulty of phase estimation, turbulence independent quantities need to be constructed.

\footnotetext{
*https://github.com/andferrari/PAINTER.jl.
} 


\subsection{Closure phases}

The closure phase allows to get rid of atmospheric effects for triplets of complex visibilities. For three baselines $\mathbf{b}_{a, b}, \mathbf{b}_{b, c}$ and $\mathbf{b}_{a, c}$ corresponding to a triplet $(a, b, c)$ of telescopes. The closure phase $(\psi)$ is defined as the phase of the bispectrum, ${ }^{4}$ i.e., the Fourier transform of the triple correlation, and is defined as:

$$
\psi_{a, b, c}^{\lambda_{n}}=\angle \mathrm{y}_{a, b}^{\lambda_{n}} \mathrm{y}_{b, c}^{\lambda_{n}} \mathrm{y}_{a, c}^{\lambda_{n}{ }^{*}}=\varphi_{a, b}^{\lambda_{n}}+\varphi_{b, c}^{\lambda_{n}}-\varphi_{a, c}^{\lambda_{n}}=\boldsymbol{h}_{a, b, c}^{\lambda_{n}} \boldsymbol{\varphi}^{\lambda_{n}}
$$

where $\boldsymbol{\varphi}^{\lambda_{n}}$ is the vector containing all unperturbed phases for wavelength $\lambda_{n}$, and $\boldsymbol{h}_{a, b, c}^{\lambda_{n}}$ is a sparse row vector with only three non zeros entries that take values $\{1,1,-1\}$. If $N_{t}$ denotes the number of telescopes, it is possible to show that $\left(N_{t}-1\right)\left(N_{t}-2\right) / 2$ independent closure phases per wavelength are available. ${ }^{5}$ In presence of turbulent measurements the phases become:

$$
\tilde{\varphi}_{a, b}^{\lambda_{n}}=\varphi_{a, b}^{\lambda_{n}}+\eta_{a}^{\lambda_{n}}-\eta_{b}^{\lambda_{n}}, \quad \tilde{\varphi}_{b, c}^{\lambda_{n}}=\varphi_{b, c}^{\lambda_{n}}+\eta_{b}^{\lambda_{n}}-\eta_{c}^{\lambda_{n}}, \quad \tilde{\varphi}_{a, c}^{\lambda_{n}}=\varphi_{a, c}^{\lambda_{n}}+\eta_{a}^{\lambda_{n}}-\eta_{c}^{\lambda_{n}},
$$

and lead to the same closure phases which are not sensible to this perturbation:

$$
\psi_{a, b, c}^{\lambda_{n}}=\tilde{\varphi}_{a, b}^{\lambda_{n}}+\tilde{\varphi}_{b, c}^{\lambda_{n}}-\tilde{\varphi}_{a, c}^{\lambda_{n}}=\varphi_{a, b}^{\lambda_{n}}+\varphi_{b, c}^{\lambda_{n}}-\varphi_{a, c}^{\lambda_{n}},
$$

\subsection{Differential phases}

For one baseline $\mathbf{b}_{a, b}$, differential phases $(\Delta \varphi)$ measure the phase evolution in wavelength with respect to a reference phase channel. The differential phases are defined by:

$$
\Delta \varphi_{a, b}^{\lambda_{k}, \lambda_{\mathrm{ref}}}=\angle \mathrm{y}_{a, b}^{\lambda_{k}}-\angle \mathrm{y}_{a, b}^{\lambda_{\mathrm{ref}}}=\varphi_{a, b}^{\lambda_{k}}-\varphi_{a, b}^{\lambda_{\mathrm{ref}}}=\boldsymbol{h}_{a, b}^{\lambda_{k}, \lambda_{\mathrm{ref}}} \boldsymbol{\varphi}
$$

The reference channel can be chosen as one of the available channels. In this case, $N_{\lambda}-1$ independent differential phases are available per baseline and $\boldsymbol{h}_{a, b}^{\lambda_{k}, \lambda_{\text {ref }}}$ is a sparse row vector with only two non zeros entries that take values $\{1,-1\}$. When the phase turbulence terms on each telescope are not independent of the wavelength $\left(\eta^{\lambda_{k}} \neq \eta^{\lambda_{\text {ref }}}\right)$ the differential phases become:

$$
\Delta \tilde{\varphi}_{a, b}^{\lambda_{k}, \lambda_{\mathrm{ref}}}=\tilde{\varphi}_{a, b}^{\lambda_{k}}-\tilde{\varphi}_{a, b}^{\lambda_{\mathrm{ref}}}=\Delta \varphi_{a, b}^{\lambda_{k}, \lambda_{\mathrm{ref}}}+\left(\eta_{a}^{\lambda_{k}}-\eta_{a}^{\lambda_{\mathrm{ref}}}\right)-\left(\eta_{b}^{\lambda_{k}}-\eta_{b}^{\lambda_{\mathrm{ref}}}\right)
$$

To a first approximation and when the spectral resolution is high enough the phase turbulence terms can be considered as slowly varying. Under this hypothesis the choice of the reference channel drive the influence of the turbulence and becomes crucial. The construction of the vector $\boldsymbol{h}_{a, b}^{\lambda_{k}, \lambda_{\mathrm{ref}}}$ is the purpose of this communication.

\subsection{Phases model}

The combination of the differential phases and phase closures into a global model improves the phase estimation: indeed, the phase closures constrain the phases of a triplet of bases at a fixed wavelength, while the differential phases constrain the phases dependence in wavelength for a given base. The constraint is illustrated in Fig. 1. To derive this model, we denote by $\boldsymbol{H}^{\boldsymbol{\Delta} \varphi}$ the matrix concatenating all vectors $\boldsymbol{h}_{a, b}^{\lambda_{k}, \lambda_{m}}$ of Eq. 5. Similarly, $\boldsymbol{H}^{\psi}=\mathbf{I}_{N_{\lambda}} \otimes \boldsymbol{H}^{\lambda_{1}}$ is a block diagonal matrix that replicates the matrix $\boldsymbol{H}^{\lambda_{1}}$, concatenating the vectors $\boldsymbol{h}_{a, b, c}^{\lambda_{n}}$ of Eq. 2. The information from the phase closures and differential phases can then be collected in a global vector $\boldsymbol{\xi}$ :

$$
\boldsymbol{\xi}=\boldsymbol{H} \varphi, \quad \boldsymbol{H}=\left[\frac{\boldsymbol{H}^{\psi}}{\boldsymbol{H}^{\Delta \varphi}}\right], \quad \boldsymbol{\xi}=\left[\frac{\psi}{\Delta \varphi}\right]
$$

where $\psi$ is the vector of all phase closures and $\Delta \varphi$ the vector of all differential phases. It follows that the phases differences, namely the differential phases and the closure phases, are jointly used to estimate the phases. Note that care must be taken about the relative power between the two phases difference observables. 


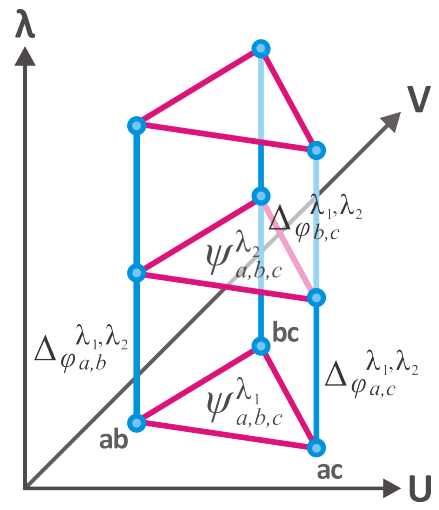

Figure 1. Illustration of the 3 dimensional constraints $(U, V, \lambda)$ for the phase estimation problem. The triplet of baselines used to construct the closure phases are represented in red and in blue the differential phase associated to each baseline.

In order to collect more data, an observation is in general repeated over time. The time laps between two observations can vary from the time needed to do a new calibration to another night of observation. When the time laps is long enough, the spatial frequencies measured during observations at a different times can be considered independent: the set of measurements of the same baseline at different wavelengths can be considered as independent over time.

The $\log$ likelihood related to the phases of the visibilities is a sum of likelihood of visibilities associated to independent bases (w.r.t the closures phase). Optimization associated to Eq. 36 in PAINTER ${ }^{1}$ which reconstructs the visibilities from the phase differences is separated in smaller optimizations. Each proximal operator reconstructs the visibilities associated to independent baselines. The splitting of the global model into sub problems allows to perform the optimization in parallel and leads to better conditioning of each sub problem.

\subsection{Dominant effect of turbulence}

In optical interferometry phases are corrupted by atmospheric turbulence. Chromatic refraction effects can be modeled by a linear combination of the three sources:

- water $(\mathrm{H} 2 \mathrm{O})^{7}$

- carbon dioxide $(\mathrm{CO} 2)$

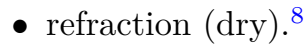

Each one is associated to a refractive index $n$ which depends on wavelength, on ambient parameters (temperature and pressure) and on its integrated quantity along the optical path. Considering the differences of ambient conditions and lengths between the interferometric beams they result in additive terms of phase drawn from data table $(\mathrm{H} 2 \mathrm{O}, \mathrm{CO} 2)$ and formulae (dry). They are illustrated in Fig. 2 for the principal observation band:

- "J": $\langle\lambda>=1.25 \mu \mathrm{m}, \Delta \lambda=0.38 \mu \mathrm{m}(\mathrm{red})$

- "H": $\langle\lambda\rangle=1.65 \mu \mathrm{m}, \Delta \lambda=0.4 \mu \mathrm{m}$ (black)

- "K": $\langle\lambda\rangle=2.2 \mu \mathrm{m}, \Delta \lambda=0.48 \mu \mathrm{m}$ (blue)

where $\langle\lambda\rangle$ and $\Delta \lambda$ are the effective wavelength and the bandwidth respectively.

The phase variations with wavelength due to these refraction effects can be considered smooth within a part of an observation band. Such additive effects can be reduced depending on the process chosen for the construction of the phase difference and is the purpose of section 3 . 

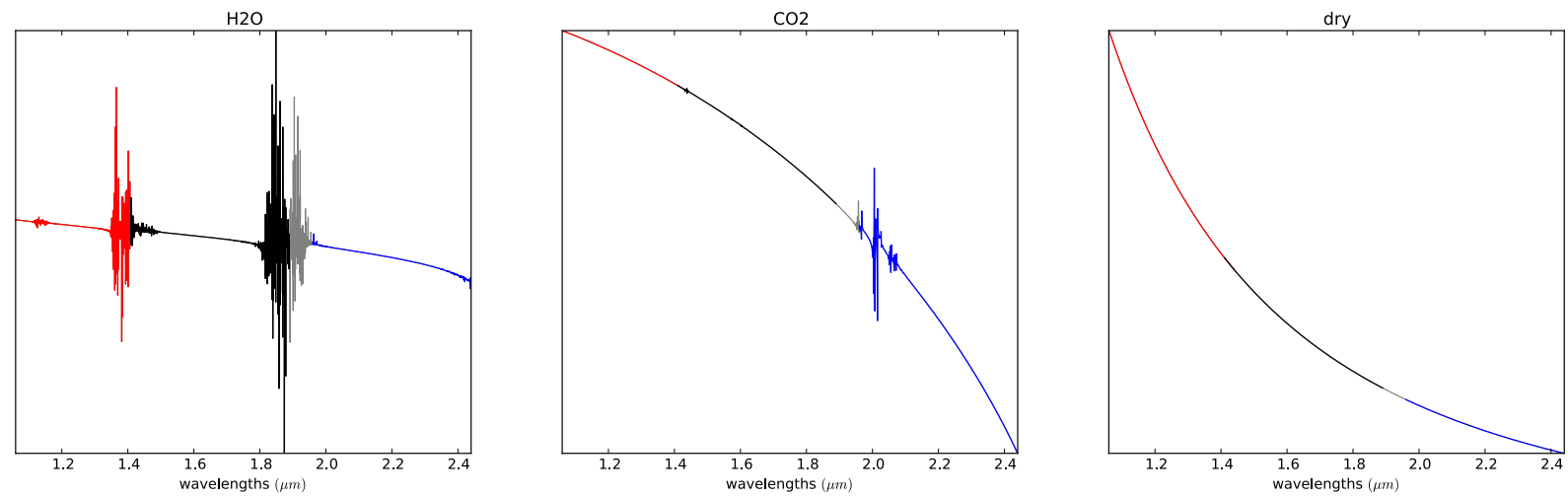

Figure 2. Example of components (H2O, CO2, dry) of atmospheric perturbation model as a function of the wavelength: Band J (red), H (black) and K (blue)

\section{PHASE TO DIFFERENTIAL PHASES MATRIX CONSTRUCTION STRATEGY}

In this section we present alternative methods to construct the differential phases. The methods are explained and illustrated on a simulated phase perturbed by main chromatic source described in section 2.4. The perturbation is generated using the dominant effect $(\mathrm{CO} 2, \mathrm{H} 2 \mathrm{O}$, refraction) with parameters fitted on real data. The resulting "noise" is applied on a simulated phase with 200 channels. Differential phase applied on phases with and without perturbation as well as the differential perturbation and the residual are shown in Fig. 6, 7 and 8.

In order to cancel the turbulence effect, the reference channel $\lambda_{\text {ref }}$ in Eq. 5 can be chosen according to different strategies.

\section{1 "Fixed" reference channel}

The "fixed" reference channel is the method originally used in PAINTER. The reference channel can be any of the channel, $\lambda_{\text {ref }}=\lambda_{m}$ :

$$
\Delta \varphi_{a, b}^{\lambda_{k}, \lambda_{m}}=\varphi_{a, b}^{\lambda_{k}}-\varphi_{a, b}^{\lambda_{m}}, \quad k \in\left[1 N_{\lambda}\right], \quad k \neq m
$$

In this case and for each base the same channel is used as reference. The resulting differential phases is constructed by subtracting this reference and is illustrated in Fig. 3 for $m$ being the first and any channel.

When a single channel is used as a reference: $\operatorname{rank}\left(\boldsymbol{H}^{\boldsymbol{\Delta} \varphi}\right)=N_{\mathbf{b}}\left(N_{\lambda}-1\right)$. In presence of atmospheric turbulence the method is illustrated in Fig. 6 with: $\lambda_{\text {ref }}=\lambda_{1}$ and $\lambda_{\text {ref }}$ chosen to be close to the maximum phase value. The effect of the turbulence is not reduced and is only changed by an offset which is its value at the reference channel.

\section{2 "Variable" reference channel}

The previous method does not cancel the long term variation of the perturbation. A way to overcome this problem is to choose a reference channel affected by a similar perturbation. A solution is to chose as a reference, $\lambda_{\text {ref }}=\lambda_{k+m}$ :

$$
\Delta \varphi_{a, b}^{\lambda_{k}, \lambda_{k+m}}=\varphi_{a, b}^{\lambda_{k}}-\varphi_{a, b}^{\lambda_{k+m}}, \quad k \in\left[1 N_{\lambda}-m\right]
$$

This case correspond to construct differential phase with phases separated by a delay $\delta \lambda$ and is illustrated in Fig 4 for a delay of 1 channel and of $m$ channels.

In presence of atmospheric turbulence this method is illustrated in Fig. 7 for a wavelength delay of 1 and 25 channels. In both cases the perturbation is substantially reduced. For $m=1$ the residual as well as the differential phases are almost null. For a greater delay the resulting differential phase is less small but the rank, is. Note that this type of reference channel induces a problem at the end of the bandwidth when $k>N_{\lambda}-m$.

When the reference channel is the "variable" reference channel: $\operatorname{rank}\left(\boldsymbol{H}^{\boldsymbol{\Delta} \varphi}\right)=N_{\mathbf{b}}\left(N_{\lambda}-m\right)$. The rank is maximum for $m=1$ which corresponds to the finite difference derivative. 


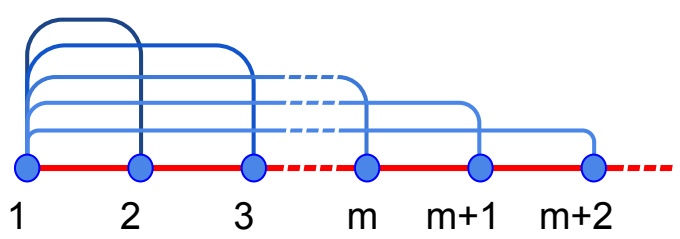

a)

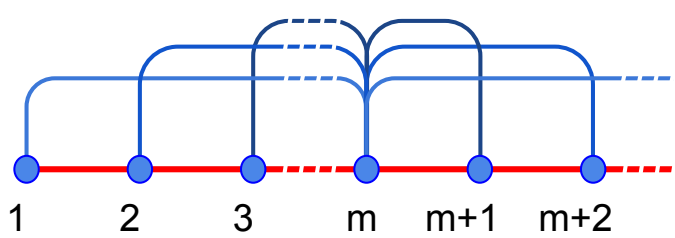

b)

Figure 3. Illustration of the construction of differential phases using the "fixed" method for one base with the reference channel being the first one $(a)$ and being the $m^{\text {th }}$ channel $(b)$

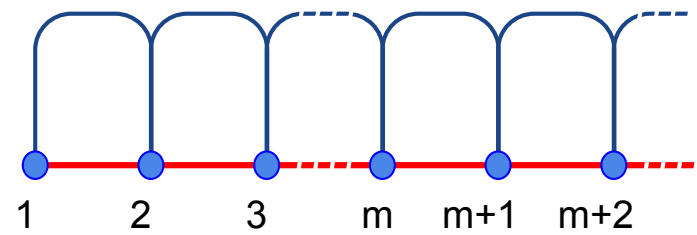

a)

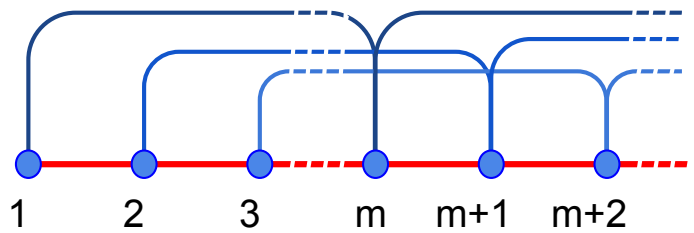

b)

Figure 4. Illustration of the construction of differential phases using the "variable" method for one base with a delay of $1(a)$ and of $m(b)$

\section{3 "Windowed" reference channel}

The "windowed" reference channel procedure divide the whole bandwidth into segments of equal length " $W_{\text {len }}$. The "fixed" reference channel method, with $m=1$, is applied separately on each segment. The windows can be contiguous or overlap as illustrated in Fig. 5.
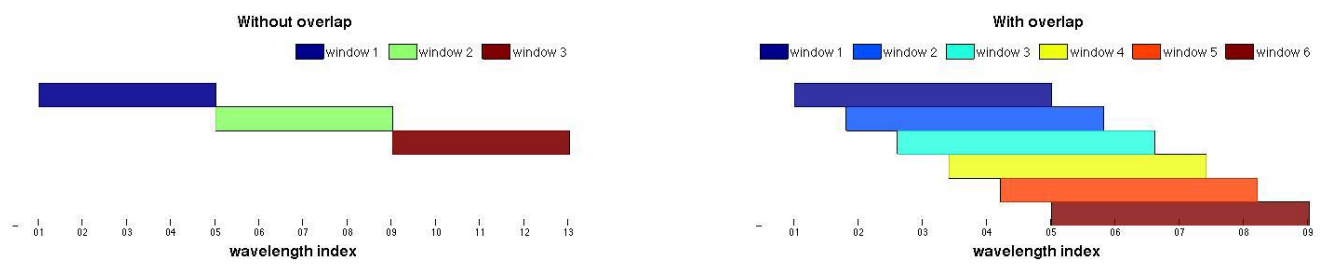

Figure 5. Left: contiguous windowing, the windows have no common channels. right: overlapping windows

- Without overlap between windows, the wavelength domain is splited into $N_{\text {win }}$ independent sub domain. As the windows have no wavelengths in common, the matrix is reduced by the number of window: $\operatorname{rank}\left(\boldsymbol{H}^{\boldsymbol{\Delta} \varphi}\right)=N_{\mathbf{b}}\left(N_{\lambda}-N_{\text {win }}\right)$.

- With an overlap of one wavelength, the first analyzed window is the same as without overlap. Each one of the following windows are translated by one wavelength and not by the length of the window. For these windows, the only non-redundant information remaining corresponds to the "variable" case with $\lambda_{\text {ref }}=\lambda_{m+W_{\text {len }}}$. This method combines the advantages of the two previous methods "fixed" and "variable". The rank of the resulting matrix is: $\operatorname{rank}\left(\boldsymbol{H}^{\Delta \varphi}\right)=N_{\mathbf{b}}\left(N_{\lambda}-1\right)$

In Fig. 8 differential phases constructed with those two methods are presented. The length of the windows is of length 25 channels in both case. On the left figure, which corresponds to a windowing without overlap, the effect of turbulence is periodically reduced. This leads to construct not smooth differential phases and the rank is not maximum in this transformation. On the right figure, the windowing with overlap leads to smoother differential phase. This last method reduces the perturbation without reducing the rank of the matrix. 

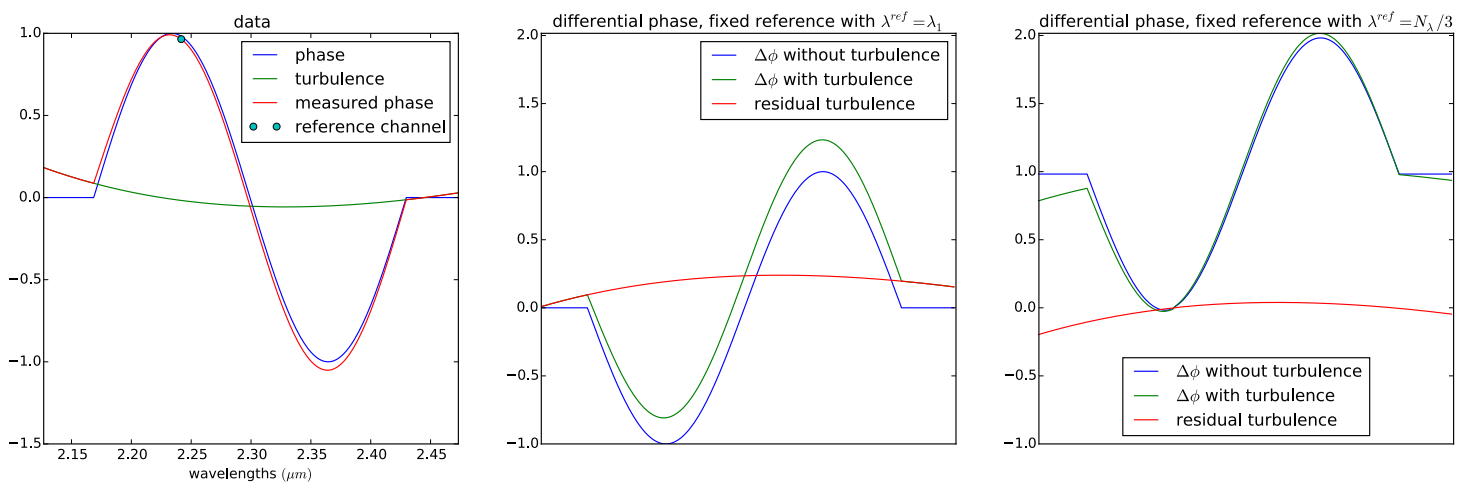

Figure 6. Example of data and turbulence in K band. Left: Phase with (red) and without (blue) perturbation, additive perturbation (green) and the reference channel (circle) used to construct the differential phase. Differential phase are constructed from a phase template with and without turbulence. Center and right: example of differential phase and residual perturbation resulting of the use of two different reference channel.
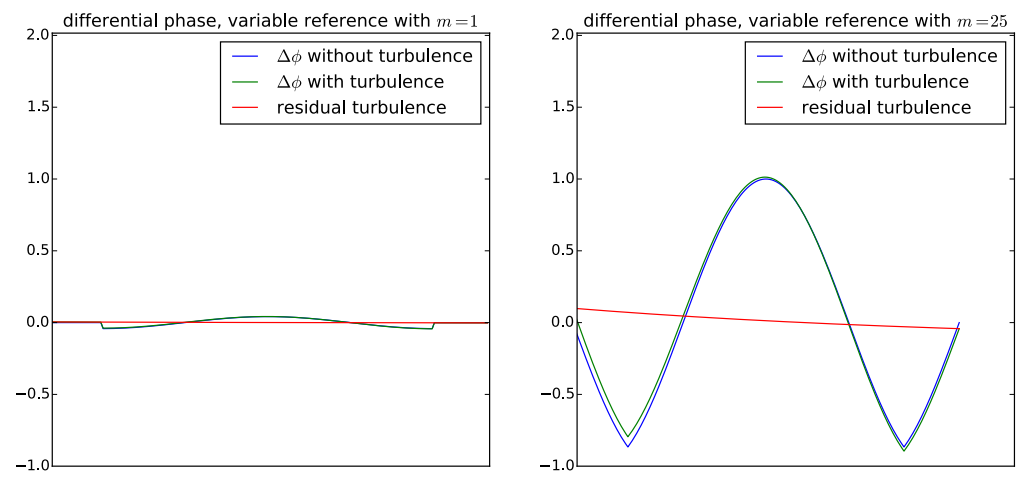

Figure 7. left and right: example of differential phase and residual perturbation resulting of the use of two different delays
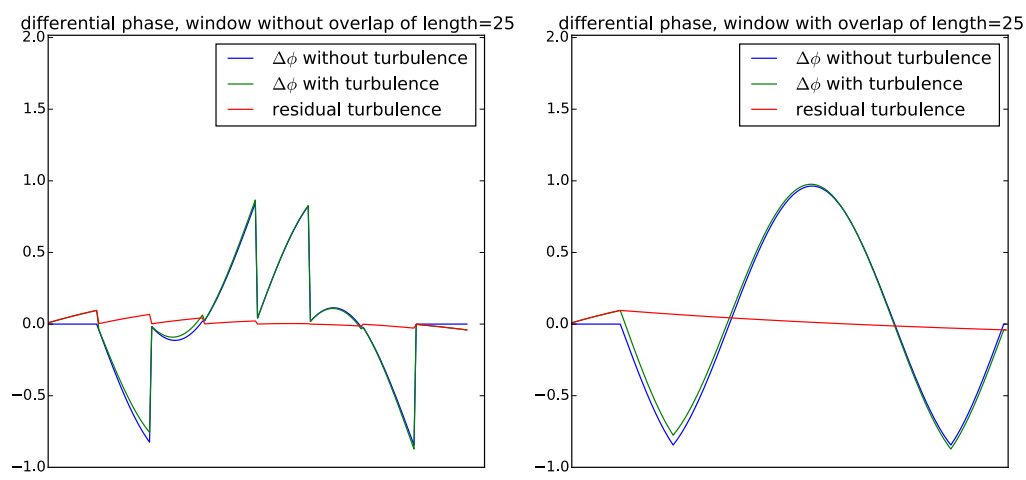

Figure 8. Left and right: example of differential phase and residual perturbation constructed with nonoverlapping/overlapping windows. 


\section{METHODS COMPARISON}

The methods with the highest rank transformation matrix namely "fixed", "variable" with $\mathrm{m}=1$ and overlapped "window" are compared. Artificial data are created and perturbed. Phases are corrupted by a linear combination of dominant atmospheric sources: $\mathrm{H} 2 \mathrm{O}$ [3], $\mathrm{CO} 2$ and dry [4] with parameters estimated on real AMBER data. PAINTER is used in same condition for all methods.

\section{1 simulated data}

The instrumental configuration is reproducing AMBER instrument and have been generated using ASPRO. ${ }^{9}$ The measurement leads to 102 bases. The model is a 3D object composed of two disks observed with a field of view of 10 mas. The first object is fixed while the position and the diameter of the second object are varying along the 100 wavelengths $(2 \mu m-2.25 \mu m)$. Some additive phases perturbation and the UV plan are shown in Fig 9 .
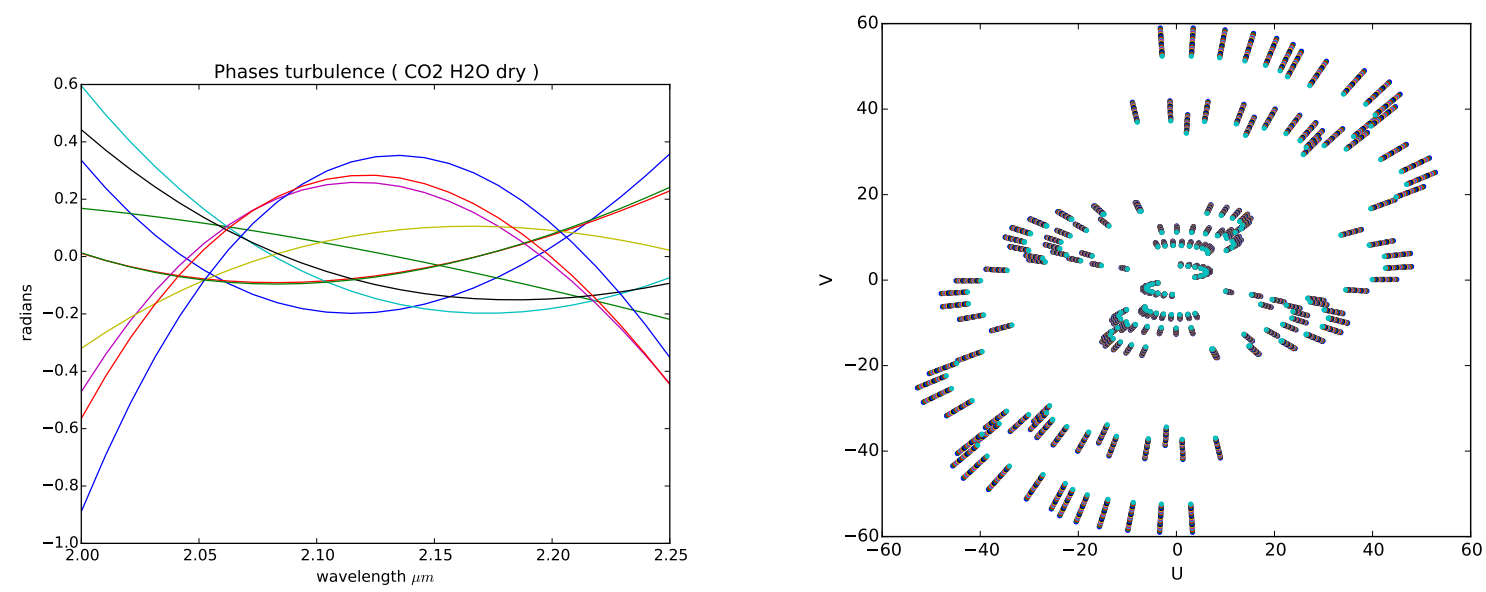

Figure 9. Left: Example of phase perturbation. Right: UV Plan.

\subsection{Results}

The model and the reconstructions are presented for the three types of reference channels: "fixed" ( $\mathrm{m}=1$ and $\left.\mathrm{m}=N_{\lambda} / 2\right)$, "window with overlap" ( $W_{\text {len }}=10$ channels) and "variable" $(\mathrm{m}=1)$ in Fig. 10 and the Signal to Noise Ratio (SNR) in dB is shown in Fig. 11:

1. the "fixed" reference channel methods suffer of long term variation whatever is the reference channel. The effect of this method on differential phases being an offset, the choice of the reference channel is not affecting the reconstruction.

2. the "variable" reference channel with a delay equal to 1 leads to a good reconstruction when the object is near symmetric. When the flux decrease, the reconstruction even with a strong spectral regularization translates the object. This is mainly due to the relative power of the differential phase to their error variance. As described $i^{1}$ the weight of differential phases are built on the sum of individual variance. As a result, the gray image obtained averaging all the images, presented on the first line of Fig. 10, does not fit the model.

3. the "windowed" reference channel with overlap gives the best results as it leads to reduced turbulence differential phases. The object are well reconstructed on all channels. The window is of length 10 channels (empirical choice), it means that the differential phases of the first 10 channels are the same for the "fixed" reference channel with $\mathrm{m}=1$. This performance can be easily compared on the SNR in Fig 11 . After the ten first channels, this methods clearly leads to the better SNR. 


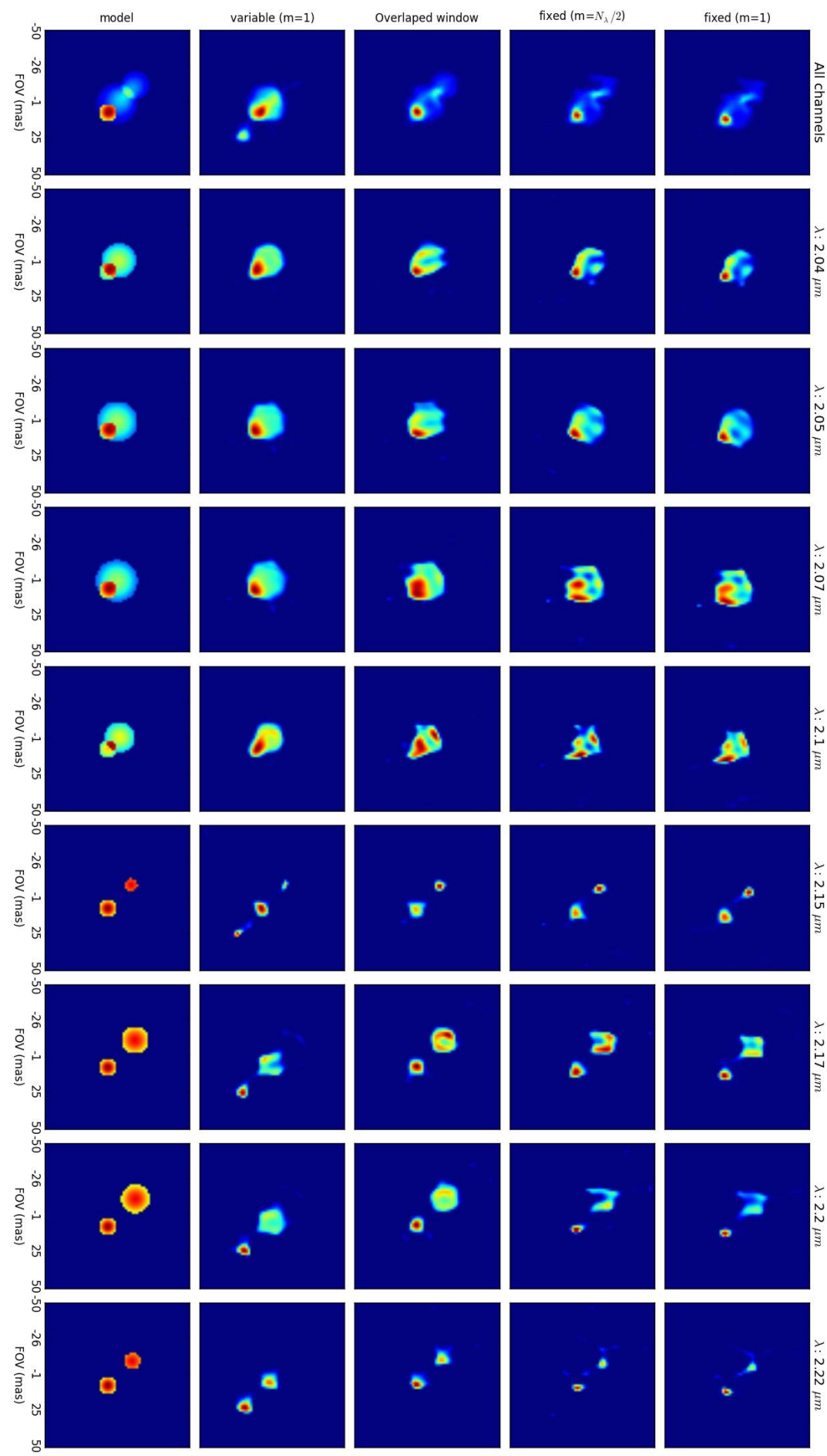

Figure 10. Model (first column) and reconstructions using PAINTER for different methods with maximum rank transformation matrix. Reconstruction are illustrated with gray images on first line and images reconstructed for some channels. 


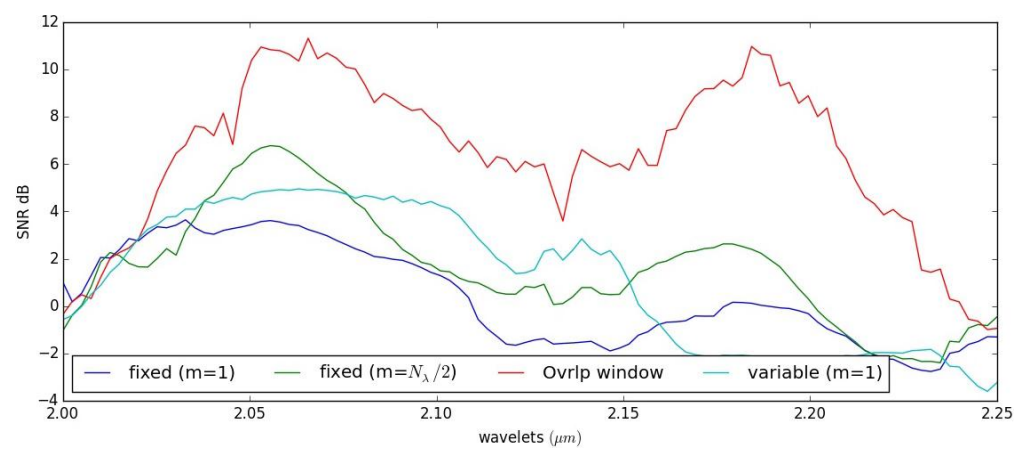

Figure 11. SNR in dB estimated on each channel for various methods.

\section{CONCLUSION AND PERSPECTIVE}

In this communication alternative methods to construct the differential phases used by PAINTER are presented, analyzed and compared on synthetic data. The results on simulated object leads to emphasis the drawback of the methods and highlight one of them as a good candidate to be systematically used. The "windowed" with overlap method leads to the best results along the whole bandwidth. Finally, note that in this study the choice of the type of reference channel does not take into account the statistics of the noise. A perspective would be to construct the reference channel in order to jointly maximize an SNR and the rank of $\boldsymbol{H}^{\Delta \varphi}$.

\section{ACKNOWLEDGMENTS}

The research leading to these results has received support from the french ANR project POLCA (ANR-2010BLAN-0511-02) and from the European Community's Seventh Framework Programme (FP7/2013-2016) under grant agreement number 312430 (OPTICON).

This work was granted access to the HPC and visualization resources of the "Centre de Calcul Interactif" hosted by University Nice Sophia Antipolis.

\section{REFERENCES}

[1] Schutz, A., Ferrari, A., Mary, D., et al., "Painter: a spatiospectral image reconstruction algorithm for optical interferometry," JOSAA 31 (Nov 2014).

[2] Schutz, A., Ferrari, A., Mary, D., Thiébaut, E., and Soulez, F., "Large scale 3d image reconstruction in optical interferometry," in [Signal Processing Conference (EUSIPCO), 2015 23rd European], 474-478 (Aug 2015).

[3] Millour, F., Meilland, A., Chesneau, O., et al., "Imaging the spinning gas and dust in the disc around the supergiant a [e] star hd 62623," A\&̈A 526 (2011).

[4] Buscher, D., "Direct maximum-entropy image reconstruction from the bispectrum," International Astronomical Union 158, 91-93 (1994).

[5] Le Besnerais, G., Lacour, S., Mugnier, L., et al., "Advanced imaging methods for long-baseline optical interferometry," Selected Topics in Signal Processing, IEEE Journal of 2, 767-780 (Oct 2008).

[6] Thiébaut, E., "Mira: an effective imaging algorithm for optical interferometry," (2008).

[7] Mathar, R. J., "Calculated refractivity of water vapor and moist air in the atmospheric window at $10 \mu \mathrm{m}$," Appl. Opt. 43, 928-932 (Feb 2004).

[8] Ciddor, P. E., "Refractive index of air: new equations for the visible and near infrared," Appl. Opt. 35, 1566-1573 (Mar 1996).

[9] Duvert, G. and Berio, P., "ASPRO: A Software to PRepare Optical interferometry observations," in [SF2A2001: Semaine de l'Astrophysique Francaise], Combes, F., Barret, D., and Thévenin, F., eds., 607 (May 2001). 